\title{
Hidalgo, C.A (2021). How Humans Judge Machines. MIT Press, Cambridge, Massachusetts, USA. ISBN: 9780262045520
}

\author{
Karamjit S. Gill ${ }^{1}$ \\ Accepted: 15 February 2021 / Published online: 25 February 2021 \\ (c) The Author(s), under exclusive licence to Springer-Verlag London Ltd. part of Springer Nature 2021
}

If you are interested in the empirical study of human-machine relations, engaged in or learning about the scenario building methodology, or are concerned about quantification of AI futures and human judgment, then this rather readable and accessible book is for you. The book takes the reader through an empirical journey of judgment, intention, and outcomes, in which people react to a variety of scenarios, and judge humans by their intentions and machines by their outcomes. On this journey, the reader is introduced to simulated scenarios ranging from algorithmic bias and fairness, privacy, displacement, and dilemmas of harms and intentions, recognizing the contextual nature of these scenarios. We learn about the direction of bias, ethics, moral dimension, perceived intention, and uncertainty of judgment. On ethics, the author takes a positivistic and empirical world-view, and focuses on 'how humans judge machines' and not 'how humans should judge machines'. In their judgment of machines, the author notes, people mistrust and express aversion to algorithmic judgments more easily than humans when they make mistakes. We are made aware of a central concern of algorithmic aversion, alerting us that we may risk rejecting technology that could improve social welfare. For example, a medical diagnostic tool that is not perfectly accurate, but is more accurate than human doctors, may be rejected if machine failures are judged or publicised with a strong negative bias. Alternatively, we may fail to recognise the consequences of technology when we show a positive bias towards algorithms.

Through scenarios ranging from self-driving vehicles, policing, facial recognition, college admissions, and human resource screening, the author discusses the way in which people judge humans and machines on issues of equity, accuracy, discrimination, bias. In scenarios on people's reaction to labour displacement in the USA, we learn that people reacted less negatively to technological displacement especially when the displacement was attributed to foreign and younger workers. It is noted that people tend to be more forgiving of machines in dilemmas that involve high levels of harm and intention and less forgiving when harm and intention are low. Whereas people are more forgiving of humans in accidental scenarios but harsher in scenarios where intention cannot be fully discarded. Building upon the multiple moral dimensions of harm, fairness, loyalty, authority, and purity, the author argues for the quantitative unpacking of ethics. In exploring this unpacking within the USA culture, it is recognized that human judgments are also culturally learnt, and may not be transferrable to other cultures. In raising a future scenario in which creative AIs collaborate with creative teams, for example, artists and directors in creative decision making, a question is raised as to how would we judge our new creative AI companions, on assigning responsibility and responsiveness? We learn about the idea of a moral space, an abstraction and a quantitative representation of moral judgment, about the idea of moral function, a mathematical object predicting how people judge the outcome of a moral scenario, the use of a statistical tool that prioritizes explicability over the accuracy, noting that this may mean designing a machine that simply mimics the moral judgment of humans in spaces such as harm, intention, intention, and wrongness.

Publisher's Note Springer Nature remains neutral with regard to jurisdictional claims in published maps and institutional affiliations.
Karamjit S. Gill

editoraisoc@yahoo.co.uk

1 University of Brighton, Brighton, UK 